\title{
Editorials
}

\section{Protamine dosing - the quandary continues}

least in some patients, lead to underdosing or overdosing with the potential for disastrous effects. By implication, the use of protamine to reverse the heparin effects should follow the same logic - i.e., the appropriate amount to reverse the effects of heparin. Too little protamine will lead to inadequate reversal of heparin effect and bleeding while too much protamine may also cause bleeding as, paradoxically, protamine has an anticoagulant effect of its own. ${ }^{7}$ Protamine is also associated with a variety of side effects, including life-threatening haemodynamic instability. ${ }^{8,9}$ These concerns have led to the measurement of heparin concentrations and employment of specific protamine titration schemes to reverse the heparin effects in a very specific manner. ${ }^{10,11}$ These investigations have demonstrated the need for increased amounts of heparin administered and reduced doses of protamine given following cardiac surgery and were associated with reductions in transfusion requirements in some ${ }^{10,11}$ but not in all ${ }^{3}$ studies. Intuitively, this seems a logical approach but the logic may be flawed. Heparin is taken up by the endothelium ${ }^{12}$ and when localised to this reservoir may be hidden from the neutralising effects of circulating protamine. Measurements of circulating heparin concentrations may not reflect the full magnitude of total body heparin concentration. Although not well characterised, the phenomenon of heparin rebound in the postoperative period is familiar to most cardiac anaesthetists and may merely reflect further mobilisation of stored heparin. Regardless of the care and specificity of the strategy employed for heparin neutralisation intraoperatively, further protamine will likely be employed as part of the overall strategy to control postoperative bleeding (summarised as follows - control of blood pressure, prolene, packed cells, platelets, plasma, protamine, and prayer). If the treatment is more protamine, what then is the value of very tight dose-titration regimens?

The logic is also predicated upon the ability of the equipment employed to measure what it says it measures 
- i.e., heparin concentration. When examined carefully this may not be so. While measurement of heparin concentrations using the Hepcon $\otimes$ system in the OR is correlated with measurements in the laboratory, ${ }_{1}^{13}$ i.e., they both measure heparin, it may not accurately measure the heparin concentration. Protamine dosing based on inaccurate information will produce inaccurate results. ${ }^{14}$

\section{The ACT is a sensitive and specific measure of protamine reversal of heparin effect}

Bull and colleagues described the use of a protamine dose titration curve based on the ACT to reverse heparin effects. ${ }^{6}$ Since then, the simplicity of the test and general applicability to the clinical situation have led to its widespread utilisation for coagulation monitoring during cardiac surgery. Before initiation of cardiopulmonary bypass (CPB), the ACT likely reflects the integrity of the haemostatic system and following heparin administration is a measure of heparin effect. ${ }^{13}$ However, after initiation of $\mathrm{CPB}$, a number of other factors come into play which may hinder the ability of the ACT to reflect the pharmacological actions of heparin. These include the duration of $\mathrm{CPB}$, residual hypothermia, the degree of fibrinolysis, haemodilution, complement activation, and the use of transfusions. ${ }^{15}$ These factors may impinge upon the coagulation system at points distinct from the site of action of heparin and, in themselves, alter the ACT. ${ }^{13}$ Certainly reductions in the ACT below commonly accepted safe levels (approximately $400 \mathrm{sec}$ ) should prompt a response including administration of heparin. However, it seems reasonable to assume that, at some point, as the duration of $\mathrm{CPB}$ continues, no matter how high the ACT, more heparin will be required ${ }^{13}$ to offset the subclinical coagulopathy associated with inadequate heparin. ${ }^{16}$ It is the author's practice to set this time limit at approximately $90 \mathrm{~min}$ (a safe minimum half-time for heparin effect $^{5}$ ) with some latitude based on the degree of hypothermia employed. Given the number of confounders present that influence the ACT, assumptions about its use as a measure by which to titrate the protamine dose seem somewhat simplistic in scope albeit reasonable for clinical practice. It is not surprising that studies using the ACT, such as that by Shore-Lesserson et al., fail to show any real differences between methods designed to produce tight control of coagulation through titration of heparin and protamine and measures which are more liberal in nature such as that based on total heparin dose.

\section{Clinical implications}

What then are the clinical implications of the study by Shore-Lesserson et al.? It would seem reasonable to suggest the following as a starting point for further discussion and study:
1 Heparin dosing before CPB should aim to achieve an ACT of $>400 \mathrm{sec}$ and ongoing monitoring of the ACT at regular intervals (30 min?) should occur. If the ACT falls below $400 \mathrm{sec}$, further supplementation of heparin dosing is required;

2 Heparin titration schemes based on heparin levels must establish that they measure heparin concentrations with acceptable sensitivity to that which occurs in the laboratory before their widespread implementation (and additional cost);

3 During $\mathrm{CPB}$ extending beyond approximately 90 min, heparin concentration is decreasing and, at some point, supplementation will be necessary regardless of the ACT;

4 Protamine dosing (whatever method is employed) should aim at the minimum necessary to reverse the heparin effect to reduce transfusion requirements and unwanted side effects. A reasonable initial endpoint is the normalisation of the ACT. Should bleeding reappear, however, additional protamine should be part of the treatment regimen.

For my part, until convinced by studies otherwise, for short perfusions of less than 90-120 min I will continue to neutralise the heparin effect using a protamine dose in a ratio of $1 \mathrm{mg}$ protamine $/ 100 \mathrm{u}$ heparin recognising that this is already an excessive dose based on the protamine neutralisation factor (PNF) indicated on the vial of heparin. However, this dosing scheme does not account for the additional heparin added to the priming solution of the CPB apparatus at the start of the perfusion $(10,000 \mathrm{u}$ in our institution). I aim to achieve a return of the ACT to the control value. If the perfusion lasts longer than $>90 \mathrm{~min}$, I supplement the heparin dose by $50 \%$ of the original dose every 90 minutes with regular monitoring of the ACT. The protamine dose for neutralisation is the same as for shorter perfusions.

\section{Dosage de la protamine - le dilemme continue}

La protamine est utilisée pour neutraliser l'héparine depuis des décades. Malgré ceci, il persiste incertitude et débat à propos du meilleur schéma posologique pour renverser les effets de l'héparine. ${ }^{1,2}$ Dans ce numéro, Shore-Lesserson et al. ajoutent au débat en comparant le système Hemochron ${ }^{\circledR} \mathrm{Rx}$ Dx de titration héparineprotamine avec le protocole standard d'administration de l'héparine à raison de $300 \mathrm{U} \cdot \mathrm{Kg}^{-1}$ avec monitorage uti- 
lisant le temps de coagulation activé (ACT) ${ }^{3}$ L'utilisation du système $\mathrm{Rx} D x$ pour titration individuelle de l'héparine ou de la protamine n'entraîne pas de réduction de la dose totale d'héparine, mais entraîne une réduction de la dose de protamine. Cependant, aucune différence entre les groupes n'a été détectée en ce qui concerne l'incidence de neutralisation incomplète de l'héparine, le rebond en héparine, les pertes par les drains médiastinaux ou les besoins en transfusions - suggérant qu'un système simple, basé sur le poids, de neutralisation de l'héparine est aussi efficace qu'un système plus sophistiqué (et coûteux) utilisant une méthode de titration. Pourquoi en est-il ainsi et quelles sont les implications de cette conclusion?

Les prérequis qui sous tendent le renversement de l'anticoagulation produite par l'héparine selon les mesures de l'ACT sont les suivantes: 1) les effets de l'héparine sont neutralisés directement par la protamine et 2) l'ACT est une mesure sensible et spécifique de la capacité de la protamine de renverser les effets de l'héparine. Examinons chacune de ces affirmations de façon critique.

\section{Renversement de l'héparine par la protamine}

Il existe une grande variabilité individuelle dans la réponse pharmacologique à une dose d'héparine. Plusieurs de nos patients cardiaques reçoivent déjà de l'héparine en pré-opératoire et le dosage de l'héparine chez cette population devient encore plus variable. ${ }^{4} \mathrm{Tel}$ qu'originalement démontré par Bull et al., ${ }^{5,6}$ ceci suggère qu'un dosage basé cliniquement sur le poids sans monitoring de l'effet anticoagulant résultant produira, au moins chez certains patients, un sous dosage ou un surdosage avec un potentiel d'effets désastreux. Par implication, l'utilisation de protamine pour renverser les effets de l'héparine devrait suivre la même logique, c'est-à-dire la quantité appropriée pour renverser les effets de l'héparine. Trop peu de protamine conduit à un renversement insuffisant avec saignement alors que trop de protamine peut aussi causer du saignement, la protamine ayant, paradoxalement, un effet anticoagulant propre. ${ }^{7}$ La protamine a aussi une variété d'effets secondaires, incluant l'instabilité hémodynamique mettant en péril la vie. ${ }^{8,9}$ Ces préoccupations ont conduit à mesurer les concentrations d'héparine et à utiliser des protocoles de titration spécifique de la protamine pour renverser l'effet de l'héparine de façon très spécifique. ${ }^{10,11}$ Ces études ont démontré la nécessité d'administrer plus d'héparine et de réduire les doses de protamine en chirurgie cardiaque, et que cette pratique était associée à une réduction des besoins transfusionnels dans certaines ${ }^{10,11}$ mais pas dans toutes les études. ${ }^{3}$ Intuitivement, cette approche semble très logique, mais cette logique peut comporter des failles. L'héparine est captée par l'endothélium ${ }^{12}$ et lorsque cachée dans ce réservoir, elle peut être cachée aux effets neutralisants de la protamine circulante. Les mesures de l'héparine circulante peuvent ne pas refléter le spectre complet de la concentration de l'héparine dans tout le corps. Bien que mal caractérisé, le phénomène du rebond d'héparine en période post-opératoire est familier à la plupart des anesthésistes cardiaques et peut refléter tout simplement la mobilisation plus complète de l'héparine emmagasinée. Indépendamment des soins et de la spécificité de la stratégie employée pour neutraliser l'héparine en période intra-opératoire, il est probable que de la protamine additionnelle sera employée dans la stratégie globale de contrôle du saignement postopératoire (résumé comme suit: contrôle de la pression, prolène, culot globulaire, plaquettes, plasma, protamine, et la prière). Si le traitement est d'administrer plus de protamine, quelle est la valeur des régimes basés sur une titration très serrée?

La logique est aussi basée sur la capacité qu'a l'équipement utilisé de mesurer ce qu'il dit qu'il mesure, c'est-à-dire la concentration d'héparine. Si on l'examine attentivement, il est possible qu'il n'en soit rien. Alors que les mesures d'héparine utilisant le système Hepcon® en salle d'opération sont correlées avec les mesures faites en laboratoire, ${ }^{13}$ i.e., les deux mesurent l'héparine, il est possible qu'il ne mesure pas précisement la concentration d'héparine. Le dosage de la protamine basé sur une information inexacte va produire des résultats inexacts. ${ }^{14}$

\section{$L^{\prime} A C T$ est une mesure sensible et spécifique de la capacité de la protamine de renverser les effets de l'béparine}

Bull et ses collègues ont décrit l'utilisation de la courbe de titration de la protamine basée sur l'ACT pour renverser les effets de l'héparine. ${ }^{6}$ Depuis, la simplicité du test et son applicabilité clinique ont conduit à son utilisation généralisée pour le monitorage de la coagulation durant la chirurgie cardiaque. Avant le début de la circulation extra-corporelle (CEC), l'ACT reflète probablement l'intégrité de l'hémostase et après l'administration de l'héparine, il mesure l'effet de l'héparine. ${ }^{13}$ Cependant, après le début de la CEC, apparaissent un nombre de facteurs qui peuvent diminuer la capacité de l'ACT à refléter les action pharmacologiques de l'héparine. Ces facteurs sont la durée de la CEC, l'hypothermie résiduelle, le degré de fibrinolyse, l'hémodilution, l'activation du complément et l'utilisation de transfusions. ${ }^{15}$ Ces facteurs peuvent perturber la coagulation à des sites différents du site d'action de l'héparine, altérant par eux-mêmes l'ACT. ${ }^{13}$ Certes, un réduction de l'ACT en deçà des valeurs sécuritaires communément acceptées (approx. $400 \mathrm{sec}$.) doivent entraîner une réponse rapide incluant l'administration d'héparine. 
Cependant, il semble raisonnable d'assumer qu'à un certain point, au moment où la $\mathrm{CEC}$ se prolonge, quel que soit le niveau de l'ACT, plus d'héparine sera requise ${ }^{13}$ pour contrer la coagulopathie sub-clinique associée à une héparinisation inadéquate. ${ }^{16} \mathrm{C}$ 'est la pratique de l'auteur de fixer cette période à environ $90 \mathrm{~min}$. (une demi-vie minimale sécuritaire pour l'héparine ${ }^{5}$ ) avec une certaine latitude basée sur le degré d'hypothermie employé. Compte tenu du nombre de facteurs présents qui influencent l'ACT, les inférences concernant son usage comme mesure de titration de la protamine semblent un peu simplistes même si c'est raisonnable pour la pratique clinique. Ce n'est pas surprenant que des études utilisant l'ACT, comme celle de Shore-Lesserson et al., ne réussissent pas à démontrer de vraies différences entre des méthodes destinées à procurer un contrôle serré de la coagulation par la titration de l'héparine et de la protamine et des méthodes plus libérales basées sur la dose totale d'héparine.

\section{Implications cliniques}

Quelles sont donc les implications de l'étude de ShoreLesserson et al? Il semble raisonnable de suggérer les éléments suivants comme point de départ pour discussions et études.

1 Le dosage d'héparine avant la CEC devrait résulter en un ACT de plus de 400 secondes et ce monitorage de l'ACT devrait être poursuivi à intervalle régulier (30 min.?). Si l'ACT tombe en bas de $\mathbf{4 0 0}$ sec. des suppléments d'héparine sont requis ;

2 Les protocoles de titration de l'héparine basés sur les taux sériques d'héparine doivent prouver qu'ils mesurent les concentrations d'héparine avec une précision comparable à celle du laboratoire avant leur implantation généralisée (et les coûts additionnels générés) ;

3 Durant la CEC de plus d'environ 90 minutes, la concentration d'héparine diminue et, à un certain point, un supplément d'héparine doit être administré indépendamment de l'ACT ;

4 Le titrage de la protamine (quelle que soit la méthode utilisée) doit viser le minimum nécessaire pour neutraliser l'effet de l'héparine de façon à réduire le saignement avec le minimum d'effets secondaires. Un but initial raisonnable est la normalisation de l'ACT. Cependant, si le saignement réapparaît, de la protamine supplémentaire doit faire partie du protocole de traitement.

Pour ma part, tant que des études ne m'auront pas convaincu du contraire, je vais continuer, lors des CEC courtes de moins de 90 à $120 \mathrm{~min}$., à neutraliser l'héparine en utilisant la protamine à raison de $1 \mathrm{mg}$ par
$100 \mathrm{U}$ d'héparine, bien conscient qu'il s'agit déjà d'une dose excessive si l'on se fie au facteur de neutralisation de la protamine (PNF) inscrit sur le flacon d'héparine. Cependant, ce mode de dosage ne tient pas compte de l'héparine ajoutée à la solution d'amorce de la CEC au moment du début de la perfusion, soit 10,000 U dans notre institution. Je vise un retour de l'ACT aux valeurs contrôle. Si la CEC dure plus de 90 minutes, j'utilise à toutes les 90 minutes un supplément d'héparine équivalent à $50 \%$ de la dose originale avec monitorage régulier de l'ACT. La dose de protamine utilisée est la même que pour les perfusions plus courtes.

\section{References}

1 Jobes DR. Pro: Tight control of anticoagulation, not empiric management, improves outcome from cardiopulmonary bypass. J Cardiothorac Anesth 1989; 3: 655-8.

$2 \mathrm{Metz}$ S. Con: Empiric management of anticoagulation, not tight control, improves outcome from cardiac surgery. J Cardiothorac Anesth 1989; 3: 659-61.

3 Shore-Lesserson L, Reich DL, DePerio M. The clinical utility of heparin and protamine dose titration in cardiac surgical patients. Can J Anaesth 1998; 45: 10-18.

4 Esposito RA, Culliford AT, Colvin SB, Thomas SJ, Lackner $H$, Spencer $F C$. Heparin resistance during cardiopulmonary bypass. The role of heparin pretreatment. J Thorac Cardiovasc Surg 1983; 85: 346-53.

5 Bull BS, Korpman RA, Huse WM, Briggs BD. Heparin therapy during extracorporeal circulation. I. Problems inherent in existing heparin protocols. J Thorac Cardiovasc Surg 1975; 69: 674-84.

6 Bull BS, Huse WM, Brauer FS, Korpman RA. Heparin therapy during extracorporeal circulation. II. The use of a dose-response curve to individualize heparin and protamine dosage. J Thorac Cardiovase Surg 1975; 69: 685-9.

7 Carr ME Jr, Carr SL. At high heparin concentrations, protamine concentrations which reverse heparin anticoagulant effects are insufficient to reverse heparin antiplatelet effects. Thromb Res 1994; 75: 617-30.

8 Horrow JC. Protamine: a review of its toxicity. Anesth Analg $1985 ; 64: 348-61$.

9 Horrow JC. Protamine allergy. J Cardiothorac Anesth $1988 ; 2: 225-42$.

10 Jobes DR, Aitken GL, Shaffer GW. Increased accuracy and precision of heparin and protamine dosing reduces blood loss and transfusion in patients undergoing primary cardiac operations. J Thorac Cardiovasc Surg 1995; 110: 36-45.

11 Despotis GJ, Joist JH, Hogue CW Jr, et al. The impact of heparin concentration and activated clotting time monitoring on blood conservation. J Thorac Cardiovasc Surg 1995; 110: 46-54. 
12 Mahadoo J, Hiebert $L$, Jaques $L B$. Vascular sequestration of heparin. Thromb Res 1977; 12: 79-90.

13 Despotis GJ, Summerfield $A L$, Joist JH, et al. Comparison of activated coagulation time and whole blood heparin measurements with laboratory plasma anti-Xa heparin concentration in patients having cardiac operations. J Thorac Cardiovasc Surg 1994; 108: 1076-82.

14 Hardy J-F, Bélisle S, Robitaille D, Perrault J, Roy $M$, Gagnon $L$. Measurement of heparin concentration in whole blood with the Hepcon/HMS device does not agree with laboratory determination of plasma heparin concentration using a chromogenic substrate for activated Factor X. J Thorac Cardiovasc Surg 1996; 112: 154-61.

15 Khuri SF, Wolfe JA, Josa $M$, et al. Hematologic changes during and after cardiopulmonary bypass and their relationship to the bleeding time and nonsurgical blood loss. J Thorac Cardiovasc Surg 1992; 104: 94-107.

16 Gravlee GP, Haddon WS, Rotbberger $H K$, et al. Heparin dosing and monitoring for cardiopulmonary bypass. A comparison of techniques with measurement of subclinical plasma coagulation. J Thorac Cardiovasc Surg 1990; 99: 518-27. 